\title{
Identification of lysosomal and extralysosomal globotriaosylceramide (Gb3) accumulations before the occurrence of typical pathological changes in the endomyocardial biopsies of Fabry disease patients
}

\author{
Ming-Jia Hsu, MS ${ }^{1}$, Fu-Pang Chang, MD ${ }^{2,3}$, Yung-Hsiu Lu, MS ${ }^{3,6}$, Sheng-Che Hung, MD $7,4,5$, \\ Yu-Chen Wang, MD², An-Hang Yang, MD², Han-Jui Lee, MD, Shih-Hsien Sung, MD ${ }^{8}$, \\ Yen-Feng Wang, MD ${ }^{9}$, Wen-Chung Yu, MD ${ }^{4}$, Ting-Rong Hsu, MD ${ }^{8}$, Po-Hsun Huang, MD ${ }^{4,9}$, \\ Sheng-Kai Chang, $\mathrm{PhD}^{6}$, Ivan Dzhagalov, PhD ${ }^{1}$, Chia-Lin Hsu, PhD ${ }^{1}$ and Dau-Ming Niu, PhD ${ }^{3,6}, 10$
}

Purpose: Evaluation standards and treatment initiation timing have been debated for a long time, particularly for late-onset Fabry disease (FD), because of its slow progression. However, early initiation of enzyme replacement therapy (ERT) for FD could be effective in stabilizing the disease progression and potentially preventing irreversible organ damage. We aimed to examine globotriaosylceramide (Gb3) deposits in patients' endomyocardial biopsies to understand the early pathogenesis of FD cardiomyopathy.

Methods: Immunofluorescent (IF) staining of Gb3 and lysosomalassociated membrane protein 1 (LAMP-1) was performed on endomyocardial biopsies of patients suspected of Fabry cardiomyopathy who had negative or only slight Gb3 accumulation determined by toluidine blue staining and electron microscopic examination.

Results: The IF staining results revealed that all patients examined had abundant Gb3 accumulation in their cardiomyocytes, including the ones who are negative for inclusion bodies. Furthermore, we found that early Gb3 deposits were mostly confined within lysosomes, while they appeared extralysosomally at a later stage.

Conclusion: A significant amount of lysosomal Gb3 deposits could be detected by IF staining in cardiac tissue before the formation of inclusion bodies, suggesting the cardiomyocytes might have been experiencing cellular stress and damage early on, before the appearance of typical pathological changes of FD during the disease progression.

Genetics in Medicine (2019) 21:224-232; https://doi.org/10.1038/ s41436-018-0010-z

Keywords: Fabry disease; Cardiomyopathy; Globotriaosylceramide; Immunofluorescent staining

\section{INTRODUCTION}

Fabry disease (FD) (MIM 301500) is an X-linked lysosomal storage disorder resulting from deficient $\alpha$ galactosidase A ( $\alpha$-Gal A) activity. ${ }^{1} \alpha-G a l$ A is an enzyme involved in the breakdown of globotriaosylceramide (Gb3). Deficient activity of this enzyme results in Gb3 accumulation in the walls of small blood vessels, nerves, dorsal root ganglia, renal glomerular and tubular epithelial cells, and cardiomyocytes. ${ }^{2}$ FD can be divided into two groups: the classic and the late-onset subtypes. ${ }^{3-6} \mathrm{New}$ born screening studies have revealed that the incidence of late-onset $\mathrm{FD}$ is much higher than that of classic FD. ${ }^{7-13}$ Affected men with classic FD exhibit little or no $\alpha-G a l$ A activity, and suffer from acroparesthesias, hypohidrosis, angiokeratomas, and often have the characteristic cornea verticillata since childhood or adolescence. ${ }^{14}$ With aging, classic FD patients develop progressive multisystemic involvements that lead to renal failure, hypertrophic cardiomyopathy, and cerebrovascular disease. ${ }^{1}$ Men with late-onset FD exhibit residual $\alpha$-Gal A activity with little or no vascular endothelial Gb3 accumulation, and lack the early clinical manifestations typical of classic FD. However, in the fourth to seventh decades of life, late-onset FD patients commonly develop cardiac disease or renal failure without skin lesions or pain. ${ }^{1,2,6,15}$

Our team revealed a high incidence $(1$ in 1,600$)$ of lateonset cardiac FD pathogenic variant, IVS4+919G $>$ A of GLA (referring to IVS4 FD), in the Taiwanese population and a

\footnotetext{
${ }^{1}$ Institute of Microbiology and Immunology, National Yang-Ming University, Taipei, Taiwan; ${ }^{2}$ Department of Pathology and Laboratory Medicine, National Yang-Ming University, Taipei, Taiwan; ${ }^{3}$ Institute of Clinical Medicine, National Yang-Ming University, Taipei, Taiwan; ${ }^{4}$ Department of Medicine, National Yang-Ming University, Taipei, Taiwan; ${ }^{5}$ Department of Biomedical Imaging and Radiological Sciences, National Yang-Ming University, Taipei, Taiwan; ${ }^{6}$ Department of Pediatrics, Taipei Veterans General Hospital, Taipei, Taiwan; ${ }^{7}$ Department of Radiology, Taipei Veterans General Hospital, Taipei, Taiwan; ${ }^{8}$ Division of Cardiology, Taipei Veterans General Hospital, Taipei, Taiwan; ${ }^{9}$ Department of Neurology, Taipei Veterans General Hospital, Taipei, Taiwan; ${ }^{10}$ Department of Medical Research, Taipei Veterans General Hospital, Taipei, Taiwan. Correspondence: C-L. Hsu (Chialin.hsu@ym.edu.tw) or D-M. Niu (dmniu1111@yahoo.com.tw)

These authors contributed equally: Ming-Jia Hsu, Fu-Pang Chang.
} 


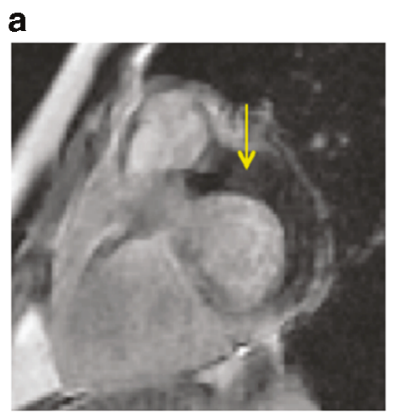

b

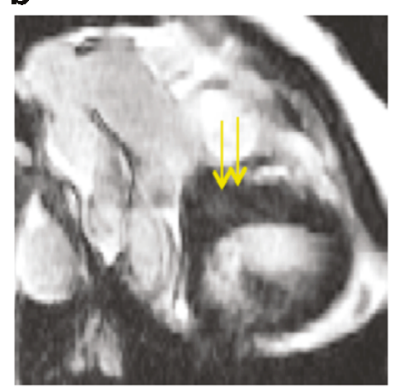

e

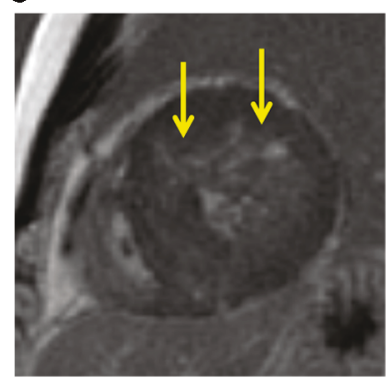

c

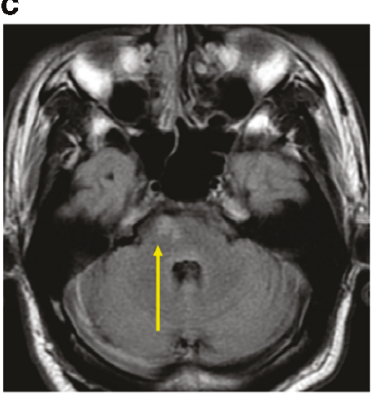

f

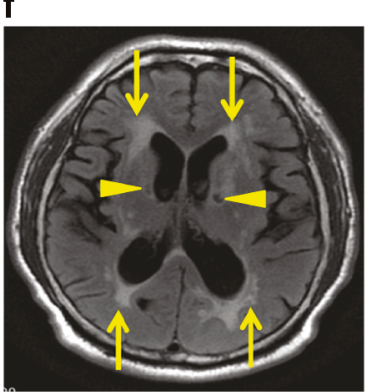

d

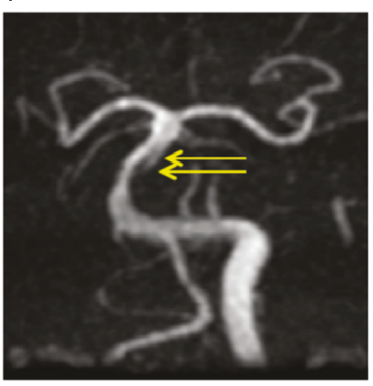

\section{.}

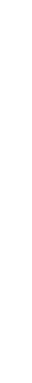

Fig. 1 Magnetic resonance image (MRI) findings of IVS4 FD patients (patients 1, 2, and 4). Gadolinium-enhanced cardiac MRI showed the late enhancements (arrows) in the left ventricle of (a) patient 1, (b) patient 2, and (e) patient 4. (c) T2-FLAIR brain MRI showed acute infarction at right ventral pons (arrow) of patient 2. (d) 3D MR angiography revealed dissection at the midbasilar artery with intimal flap (arrows) and irregular narrowing of patient 2. (f) T2 FLAIR brain MRI of patient 4 reveals old lacunar infarcts (arrowheads) and increased deep white matter hyperintensities (arrow).

number of adult patients with idiopathic hypertrophic cardiomyopathy (HCM), ${ }^{9}$ suggesting IVS4 pathogenic variant may be an unrecognized risk factor in patients with cardiac symptoms. The high prevalence of IVS4 FD in our population with unclear pathophysiology prompted the Bureau of National Health Insurance in Taiwan to institute guideline requiring IVS4 FD patients applying for enzyme replacement therapy (ERT) to confirm the primary cause of their cardiomyopathy by endomyocardial biopsy. This procedure provides us an invaluable opportunity to observe a large series of cardiopathological changes in patients with IVS4 FD with left ventricular hypertrophy (LVH). We found that all the ERT-naïve IVS4 FD patients with LVH exhibited typical histopathological changes in their cardiac biopsies (e.g., abundant diffuse cytoplasmic vacuolization in $\mathrm{H} \& \mathrm{E}$ staining and lamellar myelin bodies), ${ }^{16}$ suggesting that IVS4 pathogenic variant is a pathogenic mutation. We also discovered FD-associated cardiomyopathy can progress in silence: a significant portion of IVS4 FD patients who had developed cardiac fibrosis exhibiting neither LVH nor significant clinical signs/symptoms of cardiomyopathy. ${ }^{8}$ The endomyocardial biopsies revealed all of these patients had significant Gb3 accumulation (inclusion bodies) in their cardiomyocytes, suggesting that $\mathrm{Gb} 3$ accumulation and tissue damage might have started long before the clear signs/symptoms of cardiomyopathy.

In an attempt to initiate early intervention to prevent irreversible tissue damage, we perform endomyocardial biopsies with IVS4 FD patients who exhibited mild clinical manifestations of heart disease (detailed in the next section) but no LVH. As the number of biopsies collected in our center increased, we observed that the majority of IVS4 FD patients exhibited significant Gb3 accumulation. However, three IVS4 FD patients who were suspected of having cardiac involvement showed no signs of inclusion bodies in their biopsies by routine histopathological examinations. The 
emergence of these three patients raised two intriguing possibilities as to (1) whether the clinical signs and symptoms exhibited by these three patients were not engendered by Gb3 accumulation associated with FD, and (2) if these patients are at the early stage of cardiomyopathy progression, will these patients provide an opportunity to investigate the early pathophysiology of IVS4 FD cardiomyopathy?

To answer these questions, we aimed for a specific, sensitive, and quantitative assay to examine if there were early Fabry cardiac involvements in these patients. Previous study revealed that immune-reactive Gb3 could be detected in a classic FD patient's vascular endothelial cells that were absent of inclusions. ${ }^{17}$ Immunohistochemistry of Gb3 staining also has been successfully used to evaluate the efficacy of treatment in a Fabry mouse model whose tissue biopsies only showed mild Gb3 accumulation. ${ }^{18}$ Therefore, we applied Gb3 immunofluorescent (IF) staining to the biopsies of these patients. Two additional patients whose routine histopathological examination results showed no Gb3 or only slight Gb3 accumulation in their cardiomyocytes after more than 4 years of ERT. ${ }^{19}$ were also enrolled for comparison.

\section{SUBJECTS}

The study was conducted according to the Declaration of Helsinki and approved by the institutional review boards of Taipei Veterans General Hospital. Informed consent forms were obtained from all study subjects.

\section{Patient 1}

A 39-year-old man with IVS4 FD and family history of Fabry cardiomyopathy suffered from occasional chest pains and tightness for more than 1 year. Electrocardiography revealed complete right bundle branch block, but echocardiography showed no evidence of LVH (left ventricular mass index: $26.73 \mathrm{~g} / \mathrm{m}^{2.7}$; normal $<51$ ). His initial serum lyso-Gb3 level was $2.78 \mathrm{nM}$ (normal $<2.0 \mathrm{nM}$ ) with plasma $\alpha$-galactosidase A activity of $1.09 \mathrm{nmol} / \mathrm{h} / \mathrm{ml}$ (normal: $7.9-16.9 \mathrm{nmol} / \mathrm{h} / \mathrm{ml}$ ). Gadolinium-enhanced cardiac magnetic resonance imaging (MRI) revealed subendocardial late enhancement at the mid to basal inferior and anteroinferior lateral segments (Fig. 1a). No Gb3 accumulation was detected by H\&E and toluidine blue staining, or EM examination in his endomyocardial biopsy (Fig. 3a,b).

\section{Patient 2}

A 32-year-old man with IVS4 FD was diagnosed with acute cryptogenic stroke. Brain MRI showed an acute infarction over the right ventral pons and dissection with an intimal flap in the midbasilar artery (Fig. 1c). Mild dolichoectasia of the vertebrobasilar circulation, especially for the left vertebral artery, was also found on brain MRI (Fig. 1c,d). The plasma a-galactosidase A activity was $1.15 \mathrm{nmol} / \mathrm{h} / \mathrm{ml}$, with initial serum lyso-Gb3 level at $2.85 \mathrm{nM}$. He received an endomyocardial biopsy for the application of ERT, although he had not exhibited significant signs or symptoms of Fabry cardiomyopathy (ECG: normal, heart echo: LVMI: $46.85 \mathrm{~g} / \mathrm{m}^{2.7}$ ). The H\&E staining showed no evidence of inclusion bodies in cardiomyocytes. Unfortunately, the biopsy material submitted for EM contained no cardiomyocytic tissue, but fibroadipose tissue. The results of $\mathrm{H} \& \mathrm{E}$ staining and EM are shown in Fig. 3d,e.

\section{Patient 3}

A 36-year-old man suffered from frequent chest tightness and occasional palpitations for more than 2 years. He was identified as an IVS4 FD patient through a family study via newborn screening. His initial serum lyso-Gb3 level was 3.75 $\mathrm{nM}$, plasma a-galactosidase A activity at $1.29 \mathrm{nmol} / \mathrm{h} / \mathrm{ml}$. Although his ECG result was normal and there was no evidence of Fabry cardiomyopathy by either heart MRI or echocardiogram (LVMI: $29.46 \mathrm{~g} / \mathrm{m}^{2.7}$ ), a cardiac biopsy was performed per patient's request. No Gb3 accumulation was detected by routine examinations. The results of H\&E staining and EM are illustrated in Fig. $\mathbf{3 g}, \mathbf{h}$.

\section{Patient 4}

Patient 4 was a 61-year-old man with IVS4 FD with idiopathic cardiomyopathy for 13 years. Through high-risk screening for FD at our hospital in 2009, he was identified with IVS4 pathogenic variant. His initial serum lyso-Gb3 level before ERT was $5.19 \mathrm{nM}$. Heart MRI revealed subendocardial late enhancement at the midanterolateral, anterior, anteroseptal, apical anterior, and lateral segments (Fig. 1e). His echocardiography results showed severe concentric LVH (LVMI: $87.96 \mathrm{~g} / \mathrm{m}^{2.7}$ ). ECG showed complete right bundle branch block. His brain MRI showed old lacunar infarctions at the left centrum semiovale, bilateral corona radiata, internal capsule, lentiform nuclei, and right thalamus as well as white matter lesions at the bilateral cerebral hemisphere with generalized atrophic brain changes (Fig. 1f). He received endomyocardial biopsy after 4 years of ERT, owing to the IVS4 FD treatment guidelines in Taiwan. Gb3 accumulation was not detected in his routine cardiac biopsy examinations. The results of H\&E staining and EM are presented in Fig. $\mathbf{3 j}, \mathbf{k}$.

\section{Patient 5}

Patient 5 was a 50 -year-old man with idiopathic cardiomyopathy for 10 years. High-risk screening for FD identified this patient with IVS4 pathogenic variation. Heart MRI revealed multiple subendocardial late enhancements at the left ventricular area. Echocardiography showed moderate concentric LVH and apical hypertrophy (LVMI: $73.82 \mathrm{~g} / \mathrm{m}^{2.7}$ ). ECG showed left ventricular hypertrophy with strain. His initial serum lyso-Gb3 level prior to ERT was $6.16 \mathrm{nM}$. He received an endomyocardial biopsy after 4 years of ERT, and only slight Gb3 accumulation was detected. The results of $\mathrm{H} \& \mathrm{E}$ staining and EM are shown in Fig. $3 \mathbf{m}, \mathbf{n}$. 

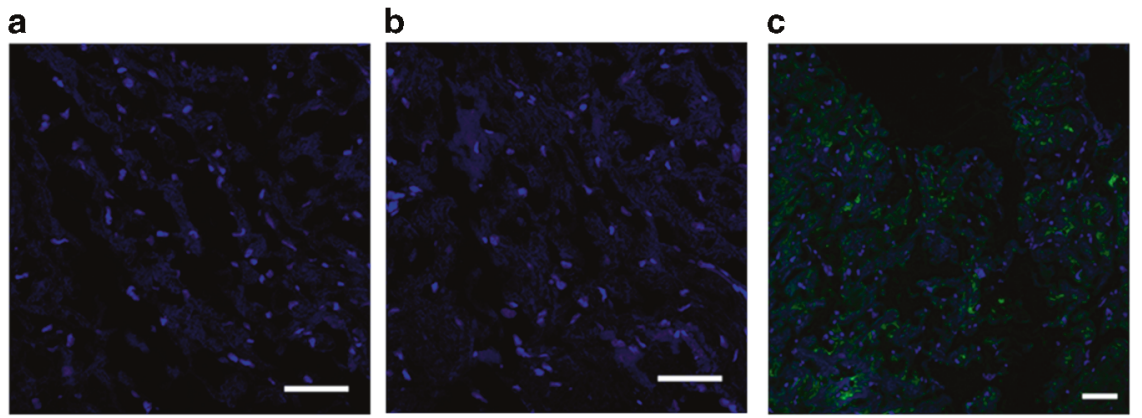

Fig. 2 Gb3 immunofluorescent staining of cardiac tissue sections. The endomyocardial biopsies were processed for Gb3 immunofluorescent staining. Both (a) secondary antibody only and (b) non-Fabry patient samples were negative for Gb3 staining, while (c) IVS4 confirmed patient showed clear signals for Gb3 staining. Representative results of three independent experiments are shown. Scale bar $=50 \mu \mathrm{m}$

\section{METHODS}

\section{Cardiac catheterization and endomyocardial biopsy}

Despite Fabry-related pathological changes in the right ventricle being typically milder than those in the left ventricle, ${ }^{9,19,20}$ right heart catheterization and right interventricular septum biopsies were chosen as methods for this study due to safety concerns. Catheterization was approached through the right internal jugular vein under digital X-ray guidance. Endomyocardial bioptome was inserted into the right ventricle, and specimens were obtained from the interventricular septum and submitted for histological examination.

\section{Routine and EM histological studies}

Cardiac specimens were fixed in $10 \%$ buffered formalin and embedded in paraffin. The sections were stained with $\mathrm{H} \& \mathrm{E}$ and Masson's trichrome and subjected to light microscopic analysis. For EM, tissues were fixed in $2.5 \%$ glutaraldehyde in phosphate buffer, postfixed with $1 \% \mathrm{OsO}_{4}$ in Sorenson's phosphate buffer, dehydrated through a graded series of ethanol washes, and subsequently embedded in Spurr's EPON. Semithin sections were prepared and stained with toluidine blue for microscopic review. Ultrathin sections were then prepared and examined through EM.

\section{Immunofluorescent staining}

Optimal cutting temperature compound (OCT) was used for embedding the cardiac tissue samples prior to frozen sectioning on a microtome-cryostat. Cardiac sections were fixed and permeabilized with a BD Cytofix/Cytoperm ${ }^{\mathrm{mm}}$ Kit (BD Biosciences), washed with BD Perm/Wash buffer, and blocked in PBS containing 5\% fetal bovine serum. The sections were incubated with anti-Gb3 antibody (gift from Dr. Sakuraba, ${ }^{21,22}$ Meiji pharmaceutical university, Japan; Tokyo Chemical Industry, clone BGR23) followed by Alexa-647 goat-anti-mouse IgG antibody (Jackson Laboratories). For the colocolization experiment, sections were stained as described above then given the additional FITCanti-human-LAMP-1 (Biolegend) antibody incubation. Hoechst was used for nuclear counterstaining. Samples were imaged through ZEISS LSM 700 confocal microscope; images were exported using Zen 2012 blue edition software (Zeiss). Gb3 and LAMP-1 immunoreactivities were shown by pseudocolor green and red precipitates, respectively. Endomyocardial biopsy from an affected IVS4 male with typical FD histopathological changes was served as positive control, while endomyocardial biopsy from a patient with cardiomyopathy other than FD was used as negative control in this study.

\section{Quantification of Gb3 accumulation by immunofluorescence signals}

To quantify the levels of Gb3 accumulation in these patients' cardiomyocytes, we applied image analysis by calculating the corrected total cell fluorescence (CTCF) of each section through ImageJ software (National Institutes of Health). The fluorescence intensity of the target protein was normalized to the number of nuclei. For each sample, at least two fields of images were collected, averaged, and subjected to CTCF calculation. Statistical analysis was performed using Prism (GraphPad). A two-tailed, unpaired, Student's $t$-test was conducted to determine the level of statistical significance.

\section{RESULTS}

Establishment and validation of Gb3 immunofluorescent staining assay

By performing the IF staining, we detected significant Gb3 staining signals in the cardiomyocytes of IVS4 FD patient biopsy with typical FD histopathological changes (inclusion bodies) (Fig. 2c, positive control). No detectable fluorescent signal was observed in the non-Fabry endomyocardial biopsy (Fig. 2b, negative control) or the biopsy staining with secondary antibody only (Fig. 2a).

Significant Gb3 accumulation observed in ERT-naïve IVS4 FD patients without typical FD histopathological findings The routine histopathological test results were negative for three ERT-naïve IVS4 FD patients, including no vacuolization in the cardiomyocytes by H\&E staining, absence of dark blue Gb3 inclusion bodies by toluidine blue staining, and no 

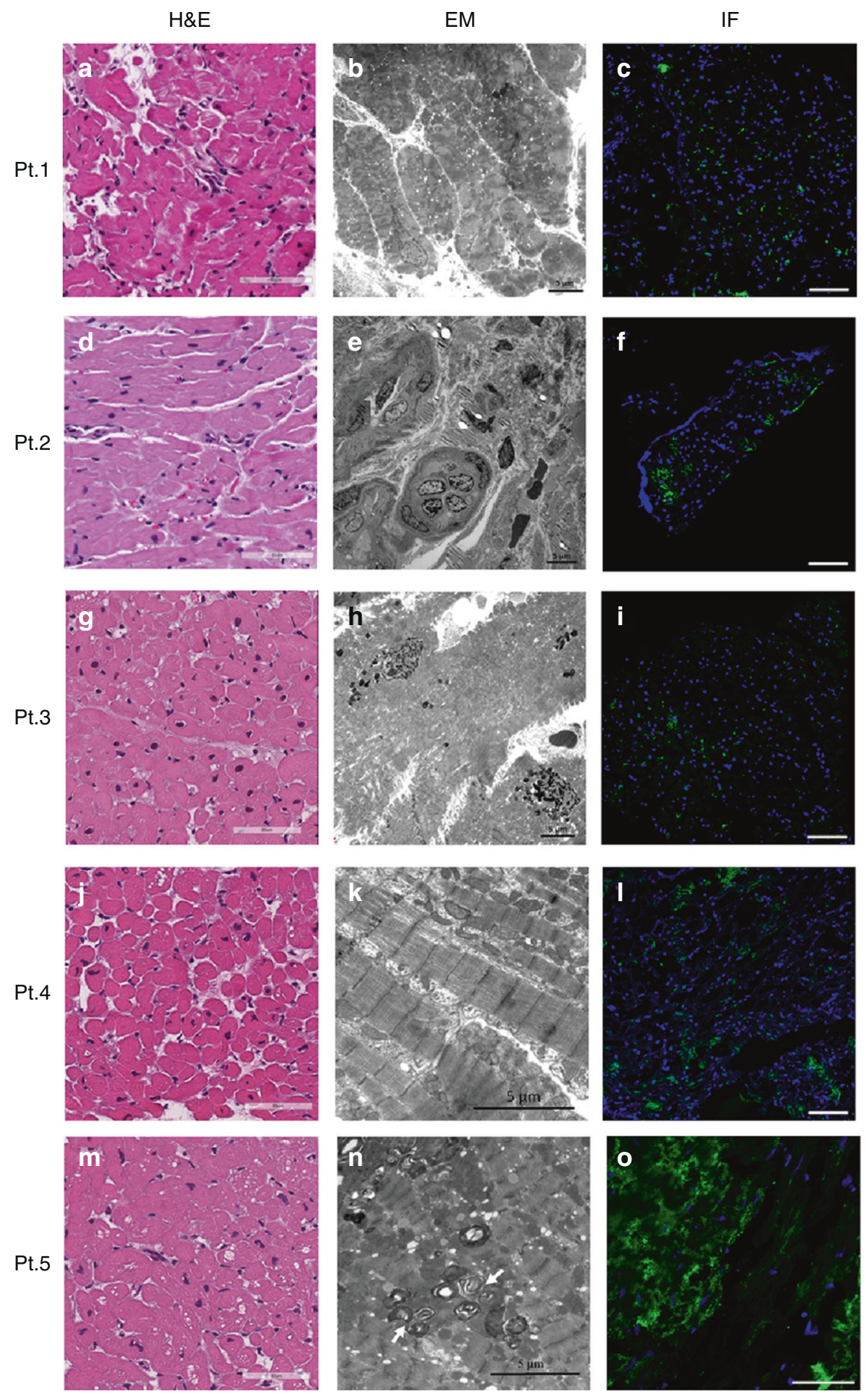

Fig. 3 The histological analysis of the IVS4 patients with no or slight Gb3 accumulation in their endomyocardial biopsies. (a, d, $g$, j, $m$ ) Routine histological analysis of H\&E staining, and (b, e, h, $\mathbf{k}, \mathbf{n}$ ) electron microscopy (EM) images showed no Gb3 inclusions in patients 1-4 and (m, $\mathbf{n}$ ) only slight Gb3 accumulation in patient 5. (e) EM of patient 2 shows only fibroadipose tissue but without cardiomyocytes in the EM specimen examined. No Gb3 inclusions can be identified in blood vessels and interstitium. (h) EM of patient 3 showed cardiomyocytes without inclusion bodies, but with a few perinuclear lipofuscin granules. (c, $\mathbf{f}, \mathbf{i}, \mathbf{l}, \mathbf{o})$ Significant $\mathrm{Gb3}$ signals could be detected in all five biopsies by immunofluorescent (IF) staining. The scale bars represented $80 \mu \mathrm{m}$ in H\&E staining, 2-5 $\mu \mathrm{m}$ in $\mathrm{EM}$ and $50 \mu \mathrm{m}$ in IF. 


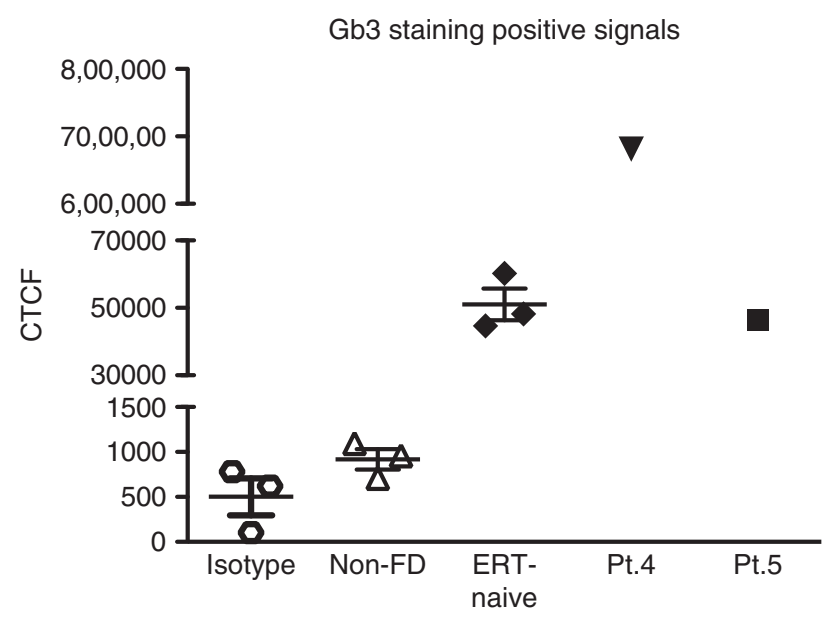

Fig. 4 Quantification of Gb3 staining fluorescence intensity. Fluorescence intensity of Gb3 immunostaining was quantified using ImageJ, and analyzed for statistical significance. Fluorescence signals of each section were quantified by calculating corrected total cell fluorescence. Tissue sections from three ERT-naiive and two ERT-treated patients were subjected to analysis. CTCF: corrected total cell fluorescence, ERT: enzyme replacement therapy, Non-FD: non-Fabry disease.

notable lamellar myelin bodies by EM. However, significant Gb3 accumulations were detected in the same endomyocardial biopsies by Gb3 IF staining (Fig. 3c,f,i).

\section{Detection of abundant residual Gb3 accumulation in ERT- treated IVS4 FD patients}

Patient 4 and 5 who had severe cardiac phenotypes and received 4 years' ERT showed no (patient 4 ) or only slight (patient 5) Gb3 inclusion bodies in the cardiomyocytes on routine (Fig. 3j,m) and EM histopathological examination (Fig. 3k,n). However, the section of the same biopsies revealed abundant Gb3 accumulation by Gb3 IF examination (Fig. 31,o).

\section{Quantification of Gb3 IF signals}

The quantified results revealed no detectable CTCF in the secondary antibody control or the non-FD tissue sections, whereas patients with IVS4 FD exhibited significantly higher $\mathrm{CTCF}$ values for Gb3 staining than control (Fig. 4). The background signals were clearly distinguishable from the positive staining, further validating the robustness of the assay. The Gb3 CTCF values of ERT-treated patients with only little Gb3 inclusion bodies had a CTCF value of approximately 700,000 ; by contrast, all the other Gb3 inclusion body negative samples had CTCF values between 40,000 and 60,000. Results from this analysis suggest that Gb3 IF is a highly sensitive assay that may provide a large window for quantification.

Distinct localization of Gb3 deposits in the cardiac tissue of ERT-naive FD patients with mild clinical manifestations The results showed two distinct Gb3 accumulation patterns in the patients examined. We observed a clear colocalization of Gb3 and LAMP-1 (Fig. 5, patients 2 and 3), suggesting that
Gb3 accumulation was confined within lysosomes in the cardiac tissue of these patients. However, we also found the combination of lysosomal and extralysosomal Gb3 accumulation patterns in the patient who had multiple late enhancements in his heart (Fig. 5, patient 1).

\section{Extralysosomal Gb3 accumulation in the ERT-treated late- onset FD patients}

In these patients we found that most of the Gb3 accumulation was present outside of the lysosomes and that the lysosomal compartments were largely free of Gb3 aggregates (Fig. 5, patients 4 and 5).

\section{DISCUSSION}

In this study, three ERT-naïve patients were found to be negative for typical FD pathological findings (inclusion body) in their cardiac tissue by routine histopathology analysis. However, significant Gb3 accumulation could be found in their cardiac biopsies by Gb3 IF staining. Gb3 was confined to lysosomes in the cardiac tissue or was found to have extralysosomal localization in the cardiomyocytes. These findings together suggest that extralysosomal Gb3 accumulation can happen before the formation of the inclusion body. Our study also revealed that Gb3 IF is a more sensitive assay to detect $\mathrm{Gb} 3$ accumulation than the routine light and $\mathrm{EM}$ histopathological examinations, and the presence of inclusion bodies by light or EM observations should not be the sole criterion for identifying disease-related storage in the heart and probably in other organs.

Besides the studies conducted in Taiwan, FD newborn screening studies from other countries have also revealed the incidence of FD to be much higher than previously expected (Italy: $1 / 3,100 ; ;^{12}$ Austria: $1 / 3,860 ;{ }^{10}$ Hungary: $1 / 13,341 ;^{13}$ Japan: $1 / 7,057 ;^{8}$ Washington, USA: $1 / 7,800 ;{ }^{11}$ Missouri, USA: $\left.1 / 2,913^{7}\right)$. These findings indicate that late-onset FD could be a critical yet latent health issue in certain ethnic populations of the world. Cardiac-related death has overtaken renal dysfunction as the most common cause of mortality in patients with $\mathrm{FD}{ }^{23,24}$ However, the underlying mechanisms responsible for FD cardiomyopathy have yet to be fully elucidated, and the most suitable time to initiate ERT to achieve more successful treatment outcomes is still under investigation. Studies have considered that the secondary effects of lysosomal glycosphingolipid accumulation such as inflammation. ${ }^{25-27}$ and circulating hypertrophy-promoting factors $^{28,29}$ could play vital roles in FD cardiomyopathy. Although the threshold of Gb3 accumulation and whether the existence of $\mathrm{Gb} 3$ deposition (inclusion) could be prerequisite factors for inducing deteriorating reactions remain unknown, the presence of Gb3 inclusion bodies in cardiac tissue is currently applied as a diagnostic standard. In this study, we argue that the appearance of $\mathrm{Gb} 3$ inclusion bodies might be a late stage sign of $\mathrm{Gb} 3$ accumulation, because Gb3 crystallization likely requires more than a saturation concentration of Gb3. Thus, we consider it highly possible that before the appearance of $\mathrm{Gb} 3$ inclusion bodies, the intracellular $\mathrm{Gb} 3$ 


\section{ARTICLE}

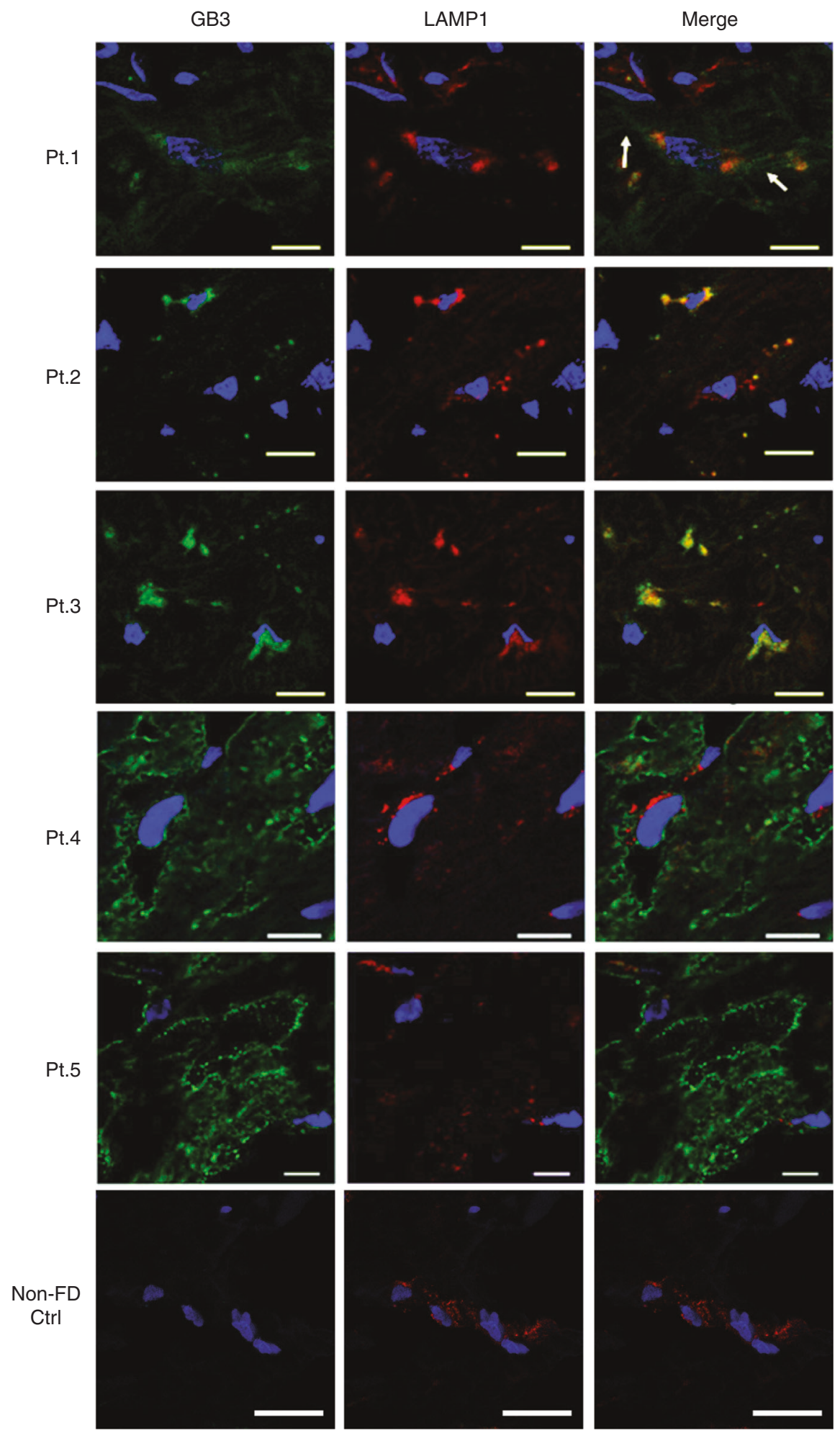

Fig. 5 Colocalization of Gb3 and LAMP-1 immunoactivity in the endomyocardial biopsies of the IVS4 patients $\mathbf{1 - 5}$. The cardiac sections of patients 1-5 and non-Fabry control were subjected to Gb3 (green), LAMP-1 (red) immunostaining. The merging of the two signals revealed strong colocalization between Gb3 and LAMP-1 in the biopsies of patients 2 and 3, but only partially in patient 1. Segregated signals of Gb3 and LAMP-1 in the biopsies of patients 4 and 5 suggest most of the Gb3 accumulation is outside the lysosomes. Scale bars $=50 \mu \mathrm{m}$. 
level might already be considerably higher than the normal level and start inducing tissue damage, although further studies are needed to provide evidence if the occult Gb3 (without inclusion body) could induce cellular stress and damages.

We speculated the presence of extralysosomal Gb3 accumulation might be a result of lysosomal rupture or leakage. The release of lysosomal Gb3 into cytosol would result in great cellular stress and impairment. ${ }^{30-32}$ This is also perhaps could be considered a late and irreversible stage of lysosome impairment. It is thus likely that the deteriorating effects of Gb3 accumulation have already been initiated in the tissues of IVS4 FD patients prior to the formation of inclusion bodies. Results from this study may imply that ERT should be initiated before the formation of $\mathrm{Gb} 3$ inclusion bodies to achieve the best treatment outcome in IVS4 FD patients. This is in agreement with a previous study that early treatment is beneficial to achieving long-term improvement in myocardial morphology, function, and exercise capacity for patients with FD with cardiomyopathy. ${ }^{33}$ Nevertheless, applying a lifelong therapy in a patient may also have significant downside, especially when there is still no strong evidence to prove that ERT begun at such an early stage will be more beneficial than ERT started after Gb3 inclusion body formation. Whether earlier initiation of ERT in life and/or prescribed for a longer time will be beneficial or detrimental calls for more thorough long-term observation studies. Although further studies with long-term follow-ups are necessary to prove this point, our study highlighted the urgent need for early detection, as well as better treatment plans for late-onset FD patients.

It is worth emphasizing that one patient already had cardiac fibrosis and complete right bundle branch block, but had no detectable Gb3 inclusion bodies in his cardiomyocytes. This observation raises the question whether "early stage" Gb3 accumulation (before the formation of Gb3 inclusion body) may already be sufficient to cause cell damage and a process that eventually causes tissue fibrosis. Tissue fibrosis is considered an irreversible event with little therapeutic intervention available, ${ }^{34,35}$ and it is regarded as a negative cardiac prognostic factor for ERT. The observation of this patient implies that severe and irreversible cardiac damage might have already occurred even in the absence of Gb3 inclusion bodies detected by routine histopathological analysis. Nevertheless, whether early-stage Gb3 accumulation (without Gb3 inclusion) can lead to the development of tissue fibrosis and its molecular mechanism remain important issues that require further investigation.

We chose to apply patient tissue biopsies as our study materials to reflect the physiological condition, however, we also recognize the caveat that the distribution of $\mathrm{Gb} 3$ accumulation might not always be homogeneous in the organ/tissue. Autopsy of patients with late-onset FD showed that Fabry-related pathological changes in the left ventricle were usually more severe than those in the right ventricle. ${ }^{36}$ Although the cardiac fibrosis of patient 1 was located in the left ventricle, only a right ventricle biopsy was performed because of safety concerns. Therefore, we cannot conclude that there were sufficient Gb3 inclusions distributed in the left ventricle to cause cardiac fibrosis in this patient.

Because the routine histopathological examination of the two patients with late-onset IVS4 FD who had received ERT for more than 4 years showed few or only low number of Gb3 inclusion bodies in the cardiomyocytes, some physicians might consider temporarily stopping or reducing the dosage/ frequency of ERT. However, our IF study showed that there were still significant levels of Gb3 accumulation in the cardiomyocytes of these patients, suggesting that regular ERT may still be required to prevent disease progression. Furthermore, it is worth noting that most of the Gb3 accumulation of these two ERT-treated patients was not localized within the lysosomes. We speculate that the abundant extralysosomal Gb3 accumulation in the cardiomyocytes might still affect the function of the cardiomyocytes of these two patients. This might be one of the reasons their cardiac function did not show significant improvement after 4 years of ERT.

The presence of fat in the heart is a phenomenon that has intrigued physicians for centuries. The cause of fatty infiltration of the heart is still not completely understood. This phenomenon is frequently found in the patients with arrhythmogenic right ventricular cardiomyopathy. However, it also could be found in other clinicopathologic groups such as myocarditis, injury, toxicity, allograft. ${ }^{37,38}$ However, current studies revealed that significant fatty infiltration could be present in the right ventricle (RV) in more than $50 \%$ of healthy elderly people. ${ }^{39}$ In our patient 2 , significant fatty infiltration was found in his endomyocardiac biopsy. It will be very interesting to understand if his fatty infiltration is related to Fabry disease or just coincident with Fabry disease.

In summary, our study suggests that Gb3 IF analysis can serve as an early detection method for patients with late-onset FD who have already started exhibiting mild symptoms, but have not yet presented detectable Gb3 accumulation in routine histological examinations. We propose to combine genomic analysis, enzyme activity testing, clinical scoring (including clinical signs and symptoms, serum lysoGb3 levels, noncontrast T1 mapping, and strain echo), and Gb3 IF staining to serve as ERT initiation guidelines for the detection of late-onset cardiac FD. Considering that patients with lateonset FD are at a high risk of developing cardiac, cerebrovascular, or neurological complications, it could be crucial to administer intervention strategies before irreversible tissue damage occurs.

\section{ACKNOWLEDGMENTS}

We would like to thank Dr. Sakuraba for his generosity in sharing the anti-Gb3 monoclonal antibody with us, and Dr. Nien-Jung Chen and Dr. Cheun-Minn Liu for insightful discussions.

This work was supported in part by the Taipei Veterans General Hospital and University System of Taiwan Joint Research Program (VGHUST105-G7-6-1, VGHUST106-G7-3-1 to C.-L. Hsu and 
D.-M. Niu) and the Ministry of Science and Technology (MOST), Taiwan (MOST-104-2323-B-010-024 to C.-L. Hsu).

\section{DISCLOSURE}

The authors declare no conflicts of interest.

\section{REFERENCES}

1. Desnick RJ, loannou YA, Eng CM. $\alpha$-Galactosidase A deficiency: Fabry disease (2001). The Metabolic and Molecular Bases of Inherited Disease. 8th edn. New York: McGraw-Hill; 2001. p. 3373-774.

2. Germain DP. Fabry disease. Orphanet J Rare Dis. 2010;5:30.

3. Bangari DS, Ashe KM, Desnick RJ, et al. alpha-Galactosidase A knockout mice: progressive organ pathology resembles the type 2 later-onset phenotype of Fabry disease. Am J Pathol. 2015;185:651-65.

4. Desnick RJ, Brady R, Barranger J, et al. Fabry disease, an underrecognized multisystemic disorder: expert recommendations for diagnosis, management, and enzyme replacement therapy. Ann Intern Med. 2003;138:338-46.

5. Nakao S, Kodama C, Takenaka T, et al. Fabry disease: detection of undiagnosed hemodialysis patients and identification of a "renal variant" phenotype. Kidney Int. 2003:64:801-7.

6. Scheidt von W, Eng CM, Fitzmaurice TF, et al. An atypical variant of Fabry's disease with manifestations confined to the myocardium. N Engl J Med. 1991;324:395-9.

7. Hopkins PV, Campbell C, Klug T, Rogers S, Raburn-Miller J, Kiesling J. Lysosomal storage disorder screening implementation: findings from the first six months of full population pilot testing in Missouri. J Pediatr. 2015;166:172-7.

8. Inoue T, Hattori K, Ihara K, Ishii A, Nakamura K, Hirose S. Newborn screening for Fabry disease in Japan: prevalence and genotypes of Fabry disease in a pilot study. J Hum Genet. 2013;58:548-52.

9. Lin $\mathrm{H}-\mathrm{Y}$, Lin HY, Chong $\mathrm{K}-\mathrm{W}$, et al. High incidence of the cardiac variant of Fabry disease revealed by newborn screening in the Taiwan Chinese population. Circ Cardiovasc Genet. 2009:2:450-6.

10. Mechtler TP, Stary S, Metz TF, et al. Neonatal screening for lysosomal storage disorders: feasibility and incidence from a nationwide study in Austria. Lancet. 2012;379:335-41.

11. Scott $C R$, Elliott $S$, Buroker $N$, et al. Identification of infants at risk for developing Fabry, Pompe, or mucopolysaccharidosis-I from newborn blood spots by tandem mass spectrometry. J Pediatr. 2013;163:498-503.

12. Spada M, Pagliardini S, Yasuda M, et al. High incidence of later-onset Fabry disease revealed by newborn screening. Am J Hum Genet. 2006:79:31-40.

13. Wittmann J, Karg E, Turi S, et al. Newborn screening for lysosomal storage disorders in Hungary. JIMD Rep. 2012;6:117-25.

14. Desnick RJ, Brady RO. Fabry disease in childhood. J Pediatr. 2004;144(5 Suppl):S20-S26.

15. Nakao S, Takenaka T, Maeda M, et al. An atypical variant of Fabry's disease in men with left ventricular hypertrophy. New Engl J Med. 1995;333:288-93.

16. Hsu TR, Hung SC, Chang FP, et al. Later onset Fabry disease, cardiac damage progress in silence: experience with a highly prevalent mutation. J Am Coll Cardiol. 2016;68:2554-63.

17. Askari $H$, Kaneski $C R$, Semino-Mora $C$, et al. Cellular and tissue localization of globotriaosylceramide in Fabry disease. Virchows Arch. 2007;451:823-34.

18. Khanna R, Soska $R$, Lun $Y$, et al. The pharmacological chaperone 1deoxygalactonojirimycin reduces tissue globotriaosylceramide levels in a mouse model of Fabry disease. Mol Ther. 2010;18:23-33.

19. Hsu T-R, Sung S-H, Chang F-P, et al. Endomyocardial biopsies in patients with left ventricular hypertrophy and a common Chinese later-onset fabry mutation (IVS4+919G A). Orphanet J Rare Dis. 2014;9:96
20. Kotani M, Kawashima I, Ozawa H, Ogura K. Generation of one set of murine monoclonal antibodies specific for globo-series glycolipids: evidence for differential distribution of the glycolipids in rat small intestine. Arch Biochem Biophys. 1994;310(1):89-96.

21. Jung SC, Han IP, Limaye A, Xu R. Adeno-associated viral vector-mediated gene transfer results in long-term enzymatic and functional correction in multiple organs of Fabry mice. Proc Natl Acad Sci USA. 2001;98: 2676-81.

22. Sakuraba H, Murata-Ohsawa M, Kawashima I, et al. Comparison of the effects of agalsidase alfa and agalsidase beta on cultured human Fabry fibroblasts and Fabry mice. J Hum Genet. 2005;51:180-8.

23. Putko BN, Wen K, Thompson RB, et al. Anderson-Fabry cardiomyopathy: prevalence, pathophysiology, diagnosis and treatment. Heart Fail Rev. 2015:20:179-91.

24. Mehta A, Clarke JTR, Giugliani R, et al. Natural course of Fabry disease: changing pattern of causes of death in FOS-Fabry Outcome Survey. J Med Genet. 2009;46:548-52.

25. Biancini GB, Vanzin CS, Rodrigues DB, et al. Globotriaosylceramide is correlated with oxidative stress and inflammation in Fabry patients treated with enzyme replacement therapy. Biochim Biophys Acta. 2012:1822:226-32.

26. De Francesco PN, Mucci JM, Ceci R, Fossati CA, Rozenfeld PA. Fabry disease peripheral blood immune cells release inflammatory cytokines: role of globotriaosylceramide. Mol Genet Metab. 2013;109:93-99.

27. Chen K-H, Chien Y, Wang K-L, et al. Evaluation of proinflammatory prognostic biomarkers for Fabry cardiomyopathy with enzyme replacement therapy. Can J Cardiol. 2016;32:1221.e1-1221.e9.

28. Barbey F, Brakch N, Linhart A, et al. Cardiac and vascular hypertrophy in Fabry disease: evidence for a new mechanism independent of blood pressure and glycosphingolipid deposition. Arterioscler Thromb Vasc Biol. 2006;26:839-44.

29. Brakch N, Dormond O, Bekri S, et al. Evidence for a role of sphingosine-1 phosphate in cardiovascular remodelling in Fabry disease. Eur Heart $J$. 2010;31:67-76

30. Griffin JL. Infantile acid maltase deficiency. Virchows Arch B. 1984;45:23.

31. Thurberg BL, Lynch Maloney C, Vaccaro C, et al. Characterization of preand post-treatment pathology after enzyme replacement therapy for Pompe disease. Lab Invest. 2006;86:1208-20.

32. Lim JA, Li L, Raben N. Pompe disease: from pathophysiology to therapy and back again. Front Aging Neurosci. 2014;6:177.

33. Beer $\mathrm{M}$, Weidemann $\mathrm{F}$, Breunig $\mathrm{F}$, et al. Impact of enzyme replacement therapy on cardiac morphology and function and late enhancement in Fabry's cardiomyopathy. Am J Cardiol. 2006:97:1515-8.

34. Kramer J, Niemann M, Störk S, et al. Relation of burden of myocardial fibrosis to malignant ventricular arrhythmias and outcomes in Fabry disease. Am J Cardiol. 2014;114:895-900.

35. Weidemann F, Niemann M, Breunig F, et al. Long-term effects of enzyme replacement therapy on Fabry cardiomyopathy: evidence for a better outcome with early treatment. Circulation. 2009;119:524-9.

36. Takenaka $T$, Teraguchi $H$, Yoshida $A$, et al. Terminal stage cardiac findings in patients with cardiac Fabry disease: an electrocardiographic, echocardiographic, and autopsy study. J Cardiol. 2008:51:50-59.

37. Basso C, Thiene G. Adipositas cordis, fatty infiltration of the right ventricle, and arrhythmogenic right ventricular cardiomyopathy Just a matter of fat?. Cardiovasc Pathol. 2005;14:37-41.

38. Kim $E$, Choe $Y H$, Han $B K$, et al. Right ventricular fat infiltration in asymptomatic subjects: observations from ECG-gated 16-slice multidetector CT. J Comput Assist Tomogr. 2007;31:22-28.

39. Fontaine $\mathrm{G}$, Fontaliran $\mathrm{F}$, Zenati $\mathrm{O}$, et al. Fat in the heart. A feature unique to the human species? Observational reflections on an unsolved problem. Acta Cardiol. 1999:54:189-94. 